Viewpoint

\title{
Role of Indigenous Healers in PHC
}

\author{
Anil Poudyal \\ BPH $23^{\text {rd }}$ Batch
}

Indigenous healer's services refer to the application of knowledge, skills, and practices based on the indigenous experiences to different cultures. These services are directed towards the maintenance of health, as well as the prevention, diagnosis, and improvement of physical and mental illness. Examples of indigenous health service providers include herbalists, faith healers, and practitioners of Chinese or Ayurvedic medicine. In contrast, allopathic service providers are those trained in western medicine. Indigenous healers play a significant role in a health system. They are usually informal, unrecognized by the government, and do not interact with the rest of the health system. Yet they can be a formal part of a system. For instance, in Nepal, there is a formal training program and a department of Ayurveda medicine within the Ministry of Health. Moreover, in China, practitioners of Chinese medicine are formally recognized.

Populations throughout Africa, Asia, and Latin America use traditional medicine to help meet this primary health care needs. In Africa, up to $80 \%$ of the population uses traditional medicine to help meet health care needs. In China, traditional medicine accounts for about $40 \%$ of all health care delivered .WHO: Traditional Medicine (2003) notes that THs are especially significant in developing countries like Nepal because they are more accessible and affordable. In addition, they are more socially accepted as compared to formally trained health workers from the urban areas .In Uganda, the prevalence of THs is 50-100 times more than that of allopathic providers. Indigenous healers are more affordable, especially for the poor. For example, in Ghana and Kenya a course of pyrimethamine/sulfodoxine antimalarials can cost several dollars, but herbal medicine for treating malaria is considerably cheaper, and the cost is often adjusted to the wealth of the patient.

Indigenous healers have also been shown to have greater leverage in treating illnesses where behavior change is needed (ie STDs) because they are often integrated and accepted in a community. Particularly, they are influential in reaching and changing the behavior of low-status, stigmatized patients, who often avoid public providers or are neglected by the public health system.

In Nepal various traditional health care practices prevalent in the community .Most commonly found practice consultation with shama healer. Populations throughout Africa, Asia, and Latin America use traditional medicine to help meet this primary health care needs.

In Uganda, the prevalence of indigenous healers is 50-100 times more than that of allopathic providers.

Indigenous healers are more affordable, especially for the poor. For example, in Ghana and Kenya a course of pyrimethamine/sulfodoxine antimalarial can cost several dollars, but herbal medicine for treating malaria is considerably cheaper, and the cost is often adjusted to the wealth of the patient. In Nepal, indigenous healers typically treat patients using various traditional techniques. These include the following treatments, used singly or in combination: phuknu (blowing evil spirit away) Tantramantra (chanting, entering a trance to wrestle with the spirits of disease),drum beating, animal sacrifice, and jadibuti (herbal medicines). Although such techniques are often criticized for being unscientific.but

recent studies have proved the effectiveness of traditional herbal medicines and chanting for relieving pain and other health problems by its hypnotic effects. These treatments are carried out using a variety of traditional tools. The most common tools include Jama (special clothes), Dhyangro (drum), Rudraksha Mala and Ghanti Bhayako Mala (garlands), Dumsi Kanda (a special cap with thorn) and Mayur ko Pwankh(feather of peacock ). More recently, indigenous healers have became interested in learning about modern medicine and using modern tools as this type of healthcare has become more available in rural Nepal.

In the developing countries, indigenous healers are an important resource who should be fully employed in the effort to provide adequate health care. They include dhami,jhankari,pandit,kabiraj etc. There are many elements in the traditional system which are beneficial while many others are not. In some South Asian countries, such as China, India and Nepal, formal training in some of these like offering courses for ensuring quality standards in the health care delivery system are part of the national health system. There are some reason why indigenous healers are more prevalent in our society .

In countries where the needs are great and the resources scarce, Lack of modern experts, inadequate proportion according to population density ,Lack of health institution, Lack of alternative resources .Lack of planning and policies formulated by organizations .Lack of health education to the people .Lack of economic opportunities.

Lack of substituting means of economy along with subsidiary. Unavailability of medicine and if available, high cost which is not affordable due to poverty. Availability of 
traditional providers which is lower cost , easily met, culturally acceptable attached. Traditional methods are acceptable due to culture and social life of the people.

In this context, indigenous healers in Nepal have a great opportunity to play an important role. The present scenario of health indicators in Nepal indicate a high death rate, a high infant mortality rate and a low contraceptive prevalence rate.The doctor-population ratio is far from accepted norms. At the same time, the main objective of the national health policy is to upgrade the health of the rural people through the primary health care approach. To reach the targets envisaged, health facilities at the different levels have been constituted.

There are some positive outcomes and changes occurs due to indigenous healers great variety of healers from different cultures were successfully trained to work in primary health care centers in Nepal. The positive outcomes are:

they were willing to work in primary health care centers ,.there is increased use of oral rehydration solution. There is rational use of treatment procedures for diarrhea.

latrines have been constructed and are in use in homes. Increasing number of cases are referred to the health centers. There is increased attendance at the health post, and traditional healers are able to act as a link between the local population and health workers. Some positive and negative role of indigenous healers are: They are easily available .They are culturally accepted and treat according to the cultural pattern of that society in which patient is living .Cheap ,Illness is psychologically removed. And some negative impact of indigenous healers are: Some of the healers use herbs and plant as therapeutic agent which unknowingly may problem. They have no scientific foundation ,evidence and logic. They can't treat physical problem (only psychological problem is treated.

In countries where the needs are great and the resources scarce, traditional healers can play a significant role in helping the rural community to improve its health and quality of life.

\section{References:}

1. World Health Organization (2003). Report on WHO Traditional Medicine Strategy 2002-2005. Geneva, World Health Organization.

2. Gartolla, Ritu Prasad,(1992):ethno medicine and other alternative medication practice .A study in medical anthropology in Nepal.

3. Bannerman, Robert H., "The Role of Traditional Medicine in Primary Health Care," Traditional Medicine and Health Care Coverage." World Health Organization, Geneva, pp 318-327, 1983.

4. World Health Organization, The promotion and development of traditional medicine. WHO, Geneva, Technical Report Series No. 622, 1978.

5. World Health Organization (2003). Report on WHO Traditional Medicine Strategy 2002-2005. Geneva, World Health Organization.

6. World Health Organization, UNICEF, Primary Health Care: Report of International Conference on Primary Health Care, Alma Ata, WHO, Geneva, 1978.

7. Ministry of Health and Population, National Health Policy 1991.MoHP,Kathmandu,1991. 